\section{FUNDING INFORMATION}

This work was supported by grants from the Swedish Research Council, Karolinska Institutet, Karolinska University Hospital, the Hesselman's Research Foundation, the Acta Oto-Laryngologica Foundation, the Nachmanssons Foundation, the Swedish Association for Otorhinolaryngology Head and Neck Surgery (SFOHH), and the European Rhinology Society (ERS).

$$
\begin{array}{r}
\text { Julia Arebro }{ }^{1,2} \text { iD } \\
\text { Cecilia Drakskog }{ }^{1} \\
\text { Ola Winqvist }{ }^{3} \\
\text { Claus Bachert } \\
\text { Sus,4 iD } \\
\text { Susanna Kumlien Georén }{ }^{1} \\
\text { Lars-Olaf Cardell }{ }^{1,2} \text { iD }
\end{array}
$$

${ }^{1}$ Division of ENT Diseases, Department of Clinical Science, Intervention and Technology, Karolinska Institutet, Stockholm, Sweden ${ }^{2}$ Department of ENT Diseases, Karolinska University Hospital, Stockholm, Sweden

${ }^{3}$ Department of Medicine, Immunology and Allergy Unit, Karolinska University Hospital, Stockholm, Sweden

${ }^{4}$ Upper Airways Research Laboratory, Ghent University, Ghent, Belgium

Correspondence

Lars-Olaf Cardell, Professor and Head of Department of ENT-Diseases, Karolinska University Hospital, 14186 Stockholm, Sweden. Email: lars-olaf.cardell@ki.se

\section{ORCID}

Julia Arebro iD https://orcid.org/0000-0002-2584-0941

Claus Bachert iD https://orcid.org/0000-0003-4742-1665

Lars-Olaf Cardell iD https://orcid.org/0000-0003-0538-9580

\section{REFERENCES}

1. Pillay J, Kamp VM, van Hoffen E, et al. A subset of neutrophils in human systemic inflammation inhibits $T$ cell responses through Mac-1. J Clin Invest. 2012;122(1):327-336.

2. Kamp VM, Pillay J, Lammers JW, Pickkers P, Ulfman LH, Koenderman L. Human suppressive neutrophils CD16bright/CD62Ldim exhibit decreased adhesion. J Leukoc Biol. 2012;92(5):1011-1020.

3. Ekstedt S, Safholm J, Georen SK, Cardell LO. Dividing neutrophils in subsets reveals a significant role for activated neutrophils in the development of airway hyperreactivity. Clin Exp Allergy. 2019;49(3):285-291.

4. Chalermwatanachai T, Vilchez-Vargas R, Holtappels G, et al. Chronic rhinosinusitis with nasal polyps is characterized by dysbacteriosis of the nasal microbiota. Sci Rep. 2018;8(1):7926.

5. Fordham MT, Mulligan JK, Casey SE, et al. Reactive oxygen species in chronic rhinosinusitis and secondhand smoke exposure. Otolaryngol Head Neck Surg. 2013;149(4):633-638.

6. Sauce D, Dong Y, Campillo-Gimenez L, et al. Reduced Oxidative Burst by Primed Neutrophils in the Elderly Individuals Is Associated With Increased Levels of the CD16bright/CD62Ldim Immunosuppressive Subset. J Gerontol A Biol Sci Med Sci. 2017;72(2):163-172.

7. Mantovani A, Cassatella MA, Costantini C, Jaillon S. Neutrophils in the activation and regulation of innate and adaptive immunity. Nat Rev Immunol. 2011;11(8):519-531.

8. Sumagin R, Robin AZ, Nusrat A, Parkos CA. Transmigrated neutrophils in the intestinal lumen engage ICAM-1 to regulate the epithelial barrier and neutrophil recruitment. Mucosal Immunol. 2014;7(4):905-915.

9. Millrud CR, Kagedal A, Kumlien Georen S, et al. NET-producing CD16(high) CD62L(dim) neutrophils migrate to tumor sites and predict improved survival in patients with HNSCC. Int $J$ Cancer. 2017;140(11):2557-2567.

\section{SUPPORTING INFORMATION}

Additional supporting information may be found online in the Supporting Information section at the end of the article.

\title{
Differential allergen expression in three Tyrophagus putrescentiae strains inhabited by distinct microbiome
}

To the Editor,

Allergen production by house dust mites is influenced directly by antibacterial compounds and digestive enzymes ${ }^{1}$ or indirectly via interactions with nutrients linked to associated microorganisms. ${ }^{1}$ Importantly, heat-stable lipopolysaccharides (endotoxins) from associated Gram-negative bacteria can affect the efficacy of allergen immunotherapy sera produced from house dust mites. ${ }^{1,2}$ House dust mites serve as carriers of bacteria, and it is possible that these bacteria are responsible for the induction of IgE sensitization to microbial antigens. ${ }^{3}$ For this reason, the microbiomes of allergen-producing mites have recently become the subject of intensive research. ${ }^{4}$ Unfortunately, the existence of variability in microbiome composition among different mite populations or strains is insufficiently considered (Erban et al, ${ }^{5}$ Lee et $\mathrm{al}^{6}$ ), despite the evidence that different strains of mites may harbor different and persistent microbial communities. In Tyrophagus putrescentiae, for example, these communities include the following bacteria: Wolbachia, Cardinium, Solitalea, Blattabacterium-like (intracellular), Bartonella-like, and Bacillus sp. (gut-associated). ${ }^{5}$ These observations open the question of whether mite strains harboring different bacterial communities differ in allergen production. 


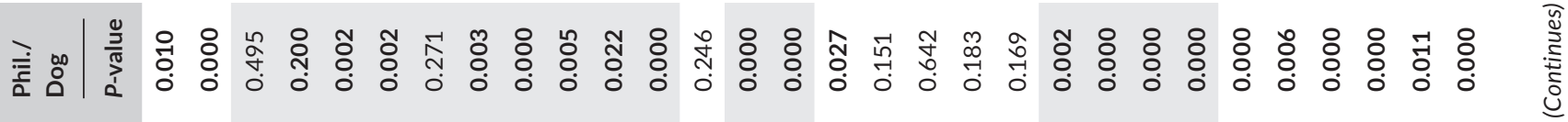

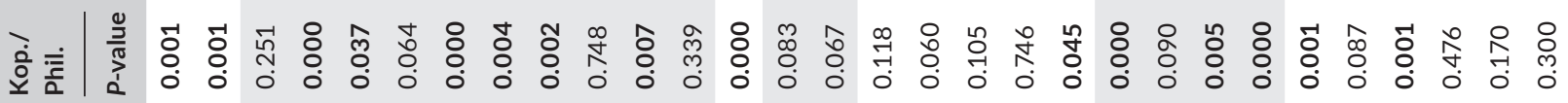

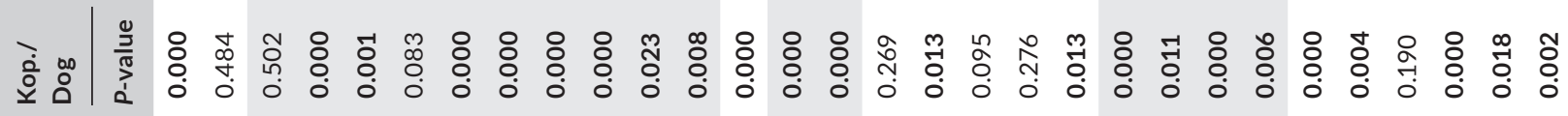

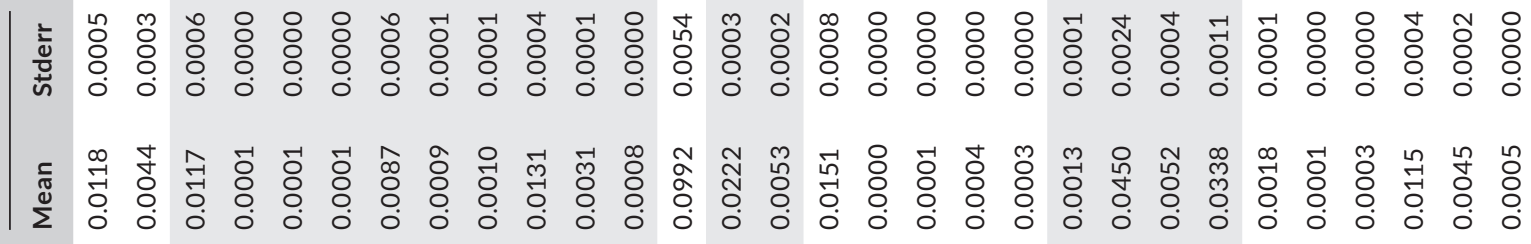

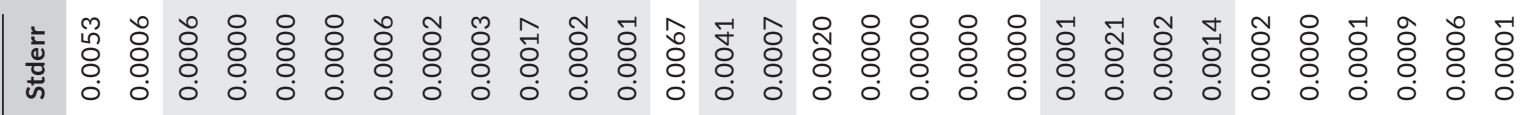

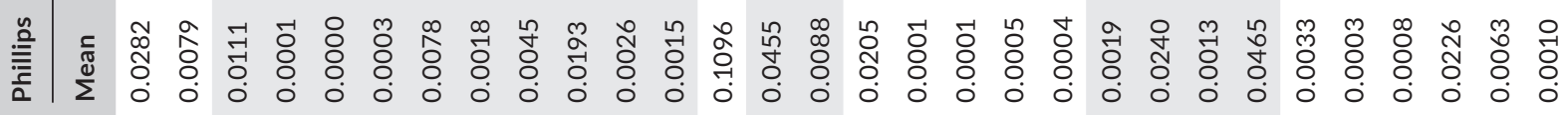

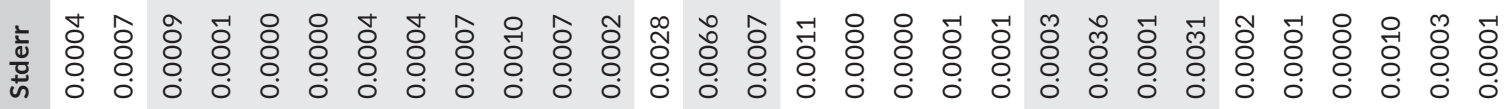

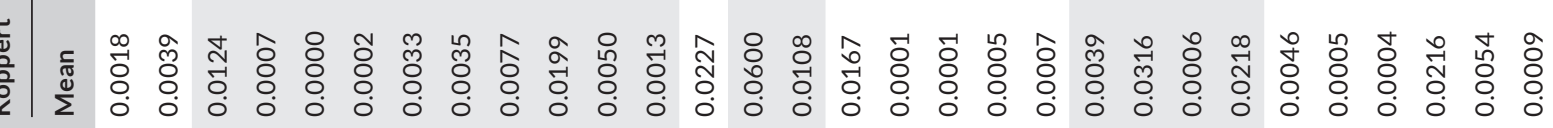

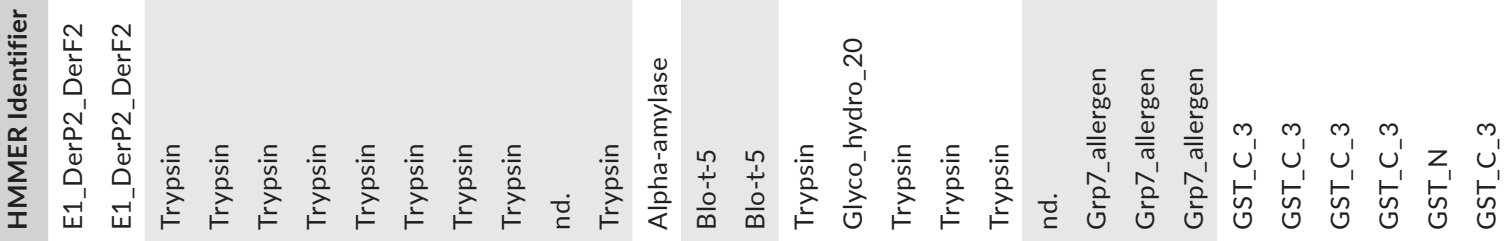

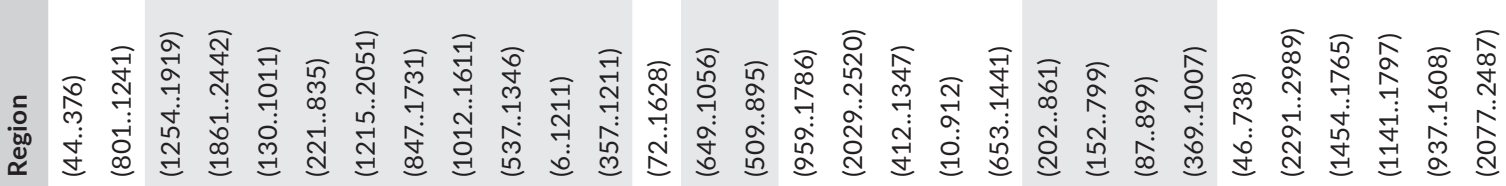

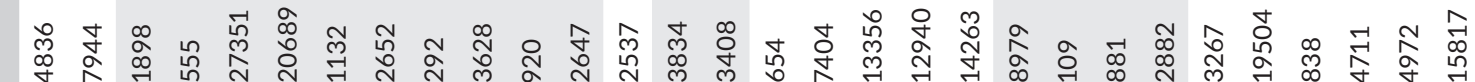

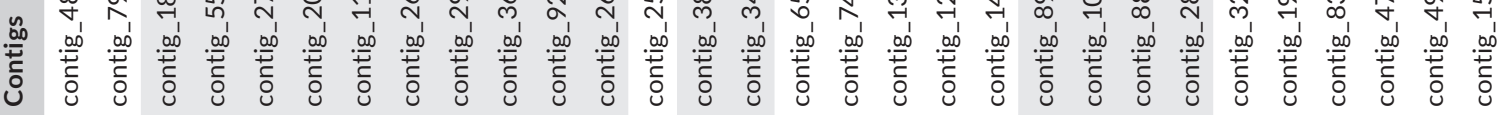

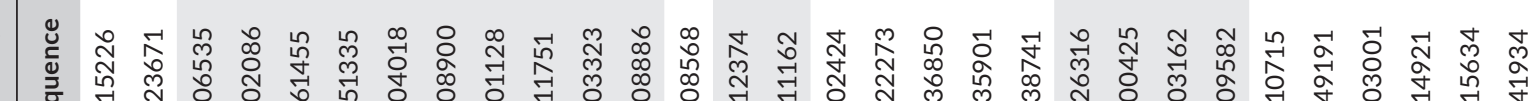

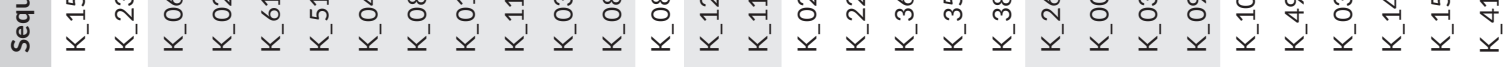
4
0
$\frac{1}{0}$
$\frac{1}{4}$
0
$\frac{1}{2}$
$\frac{0}{x}$
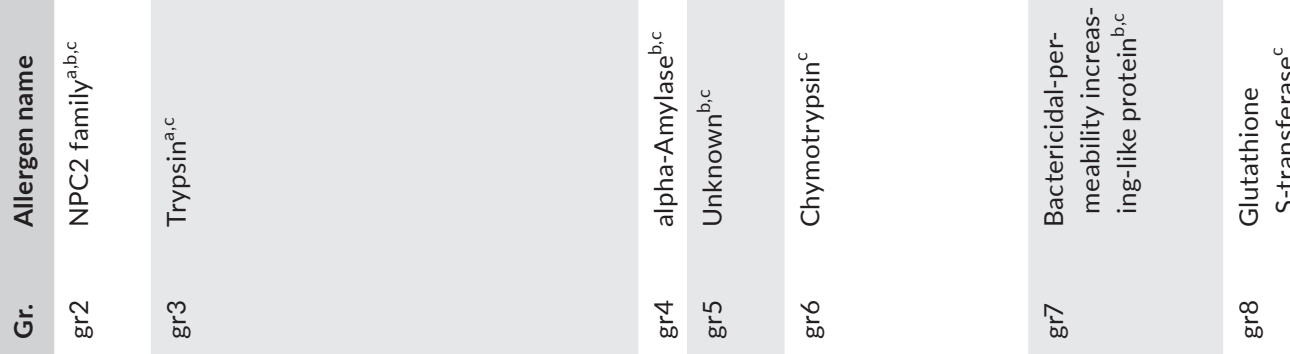


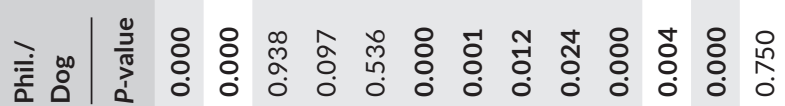

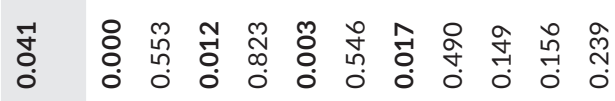

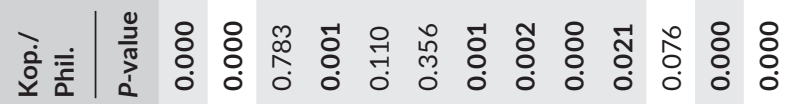

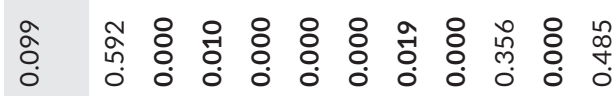

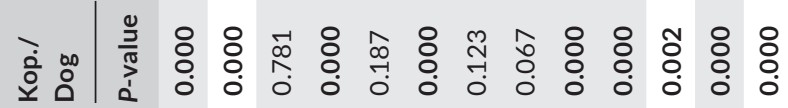

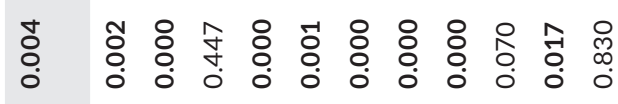

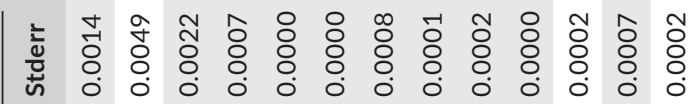

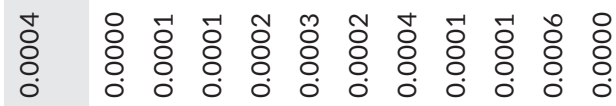

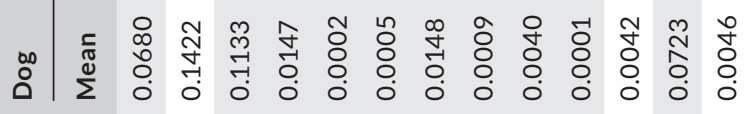

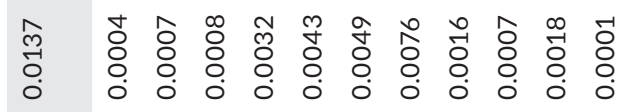

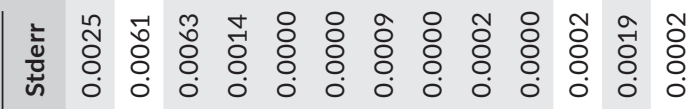

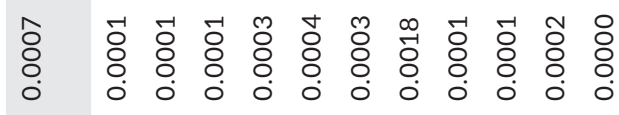

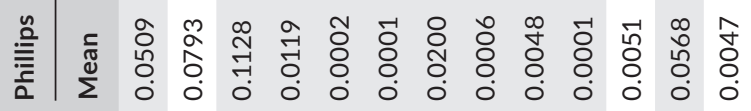

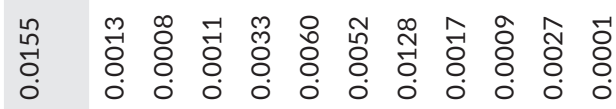

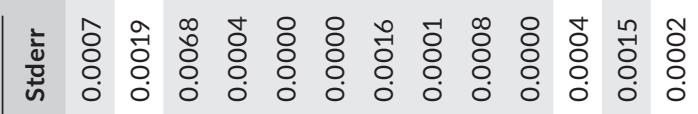

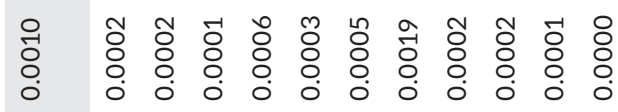

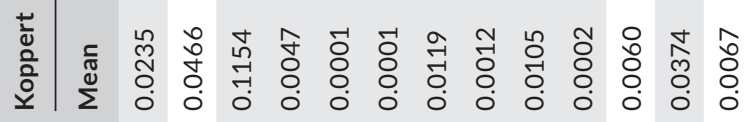

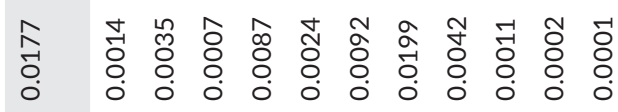

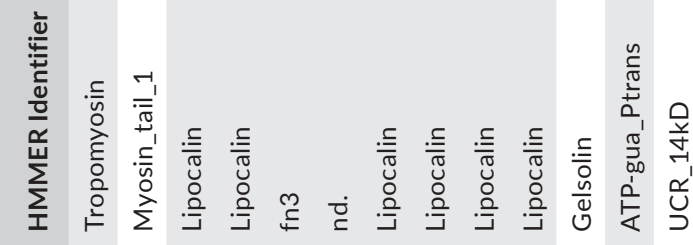

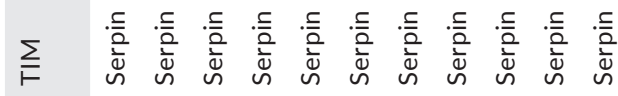

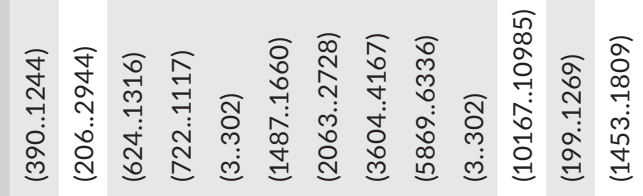

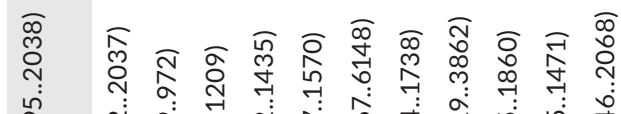

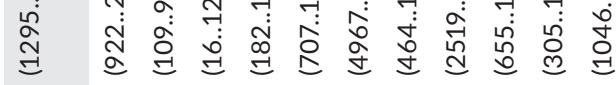

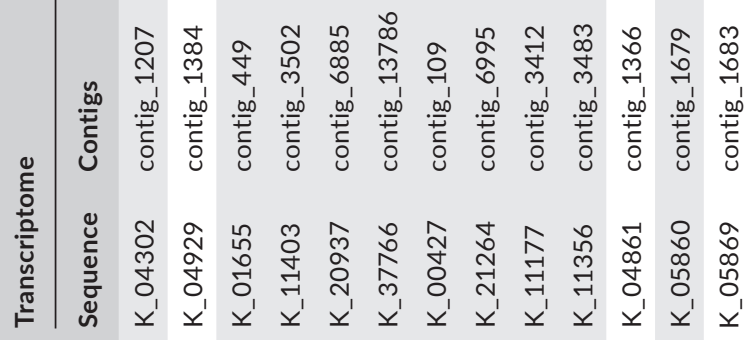

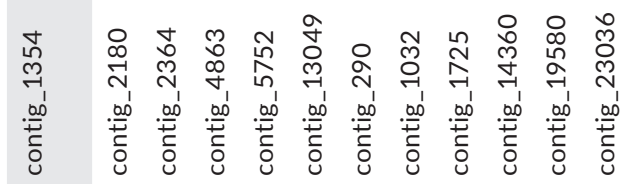

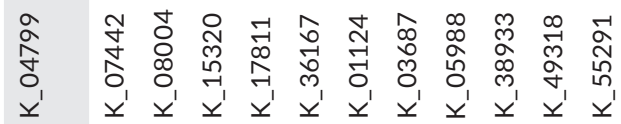

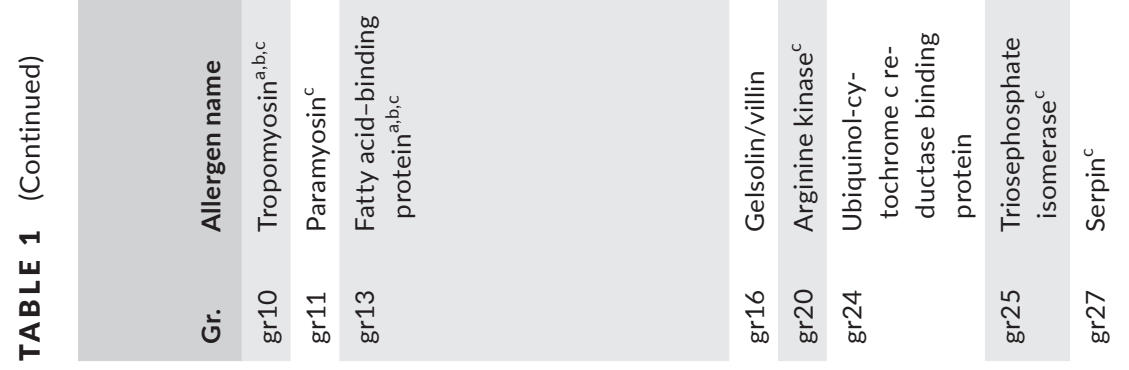




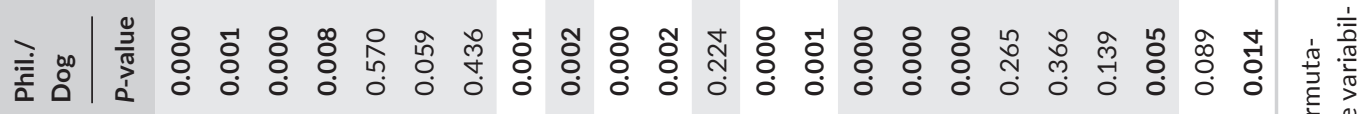

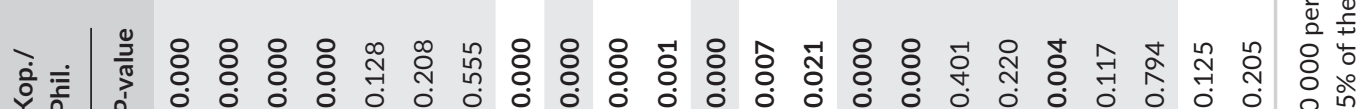

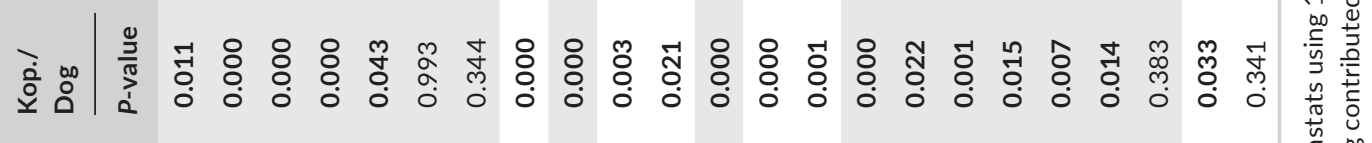

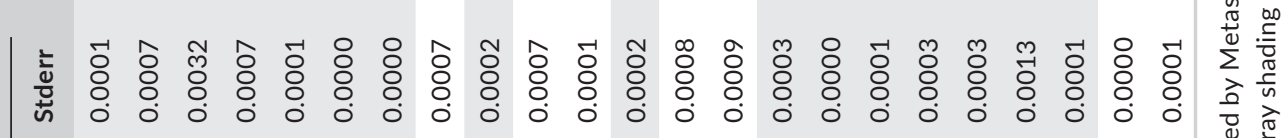

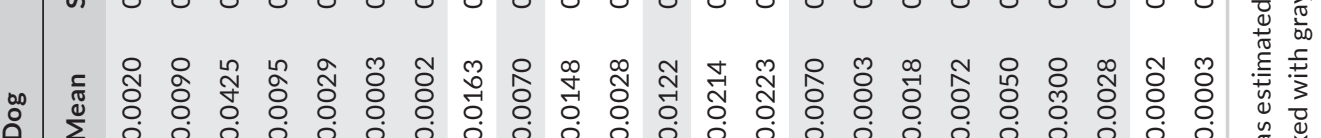

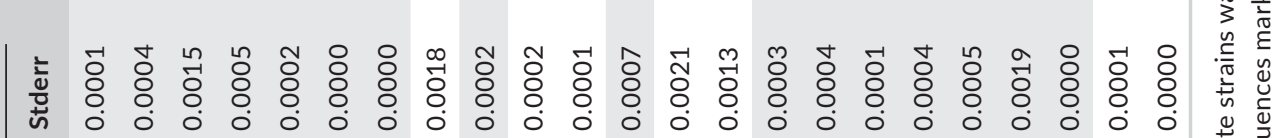

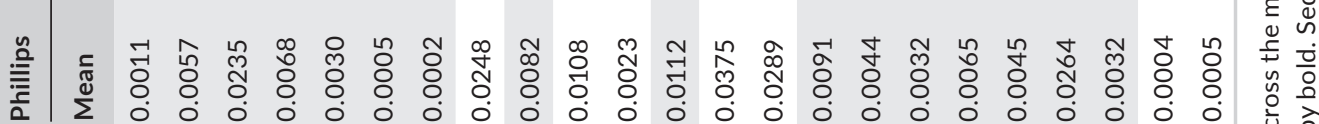

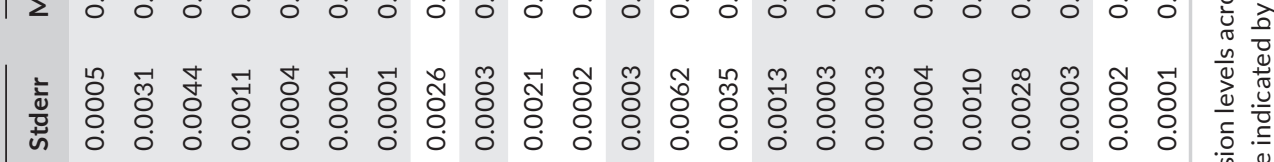

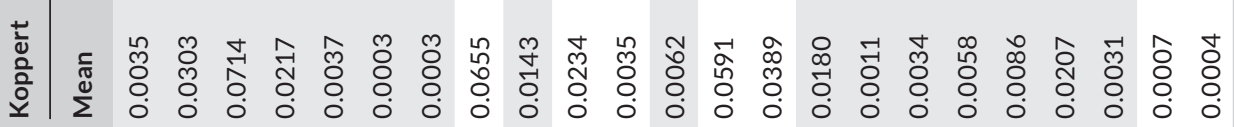

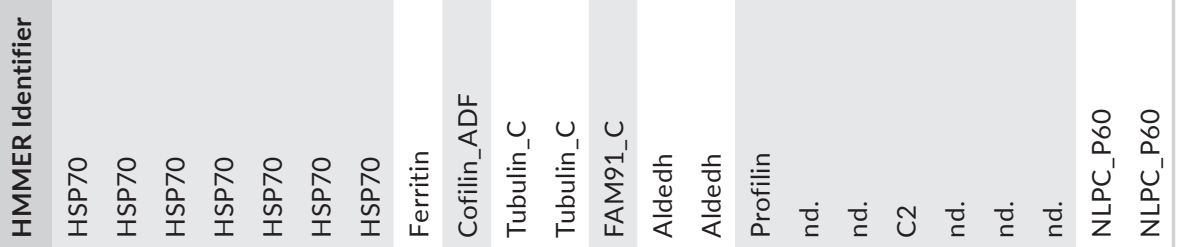

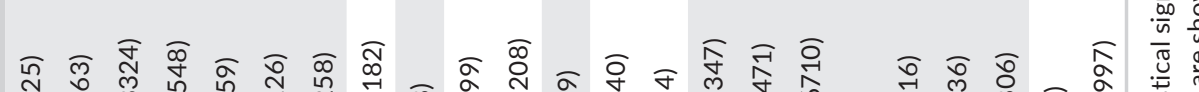

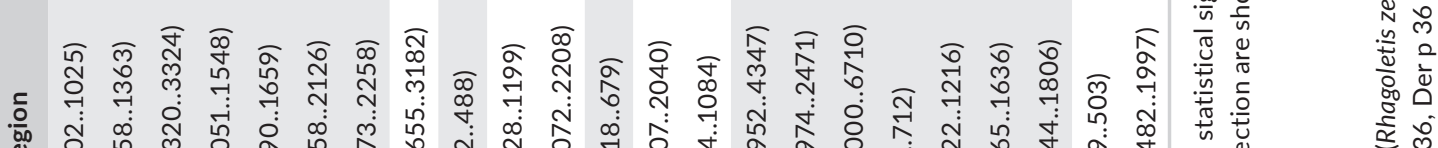

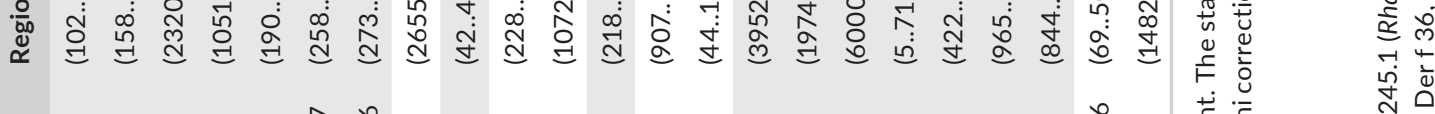

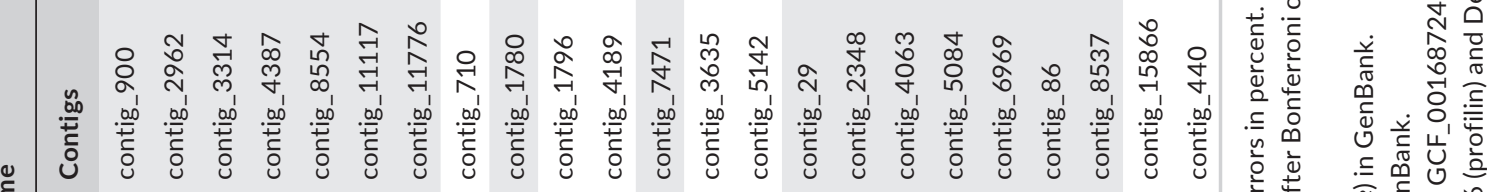

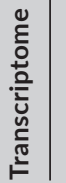

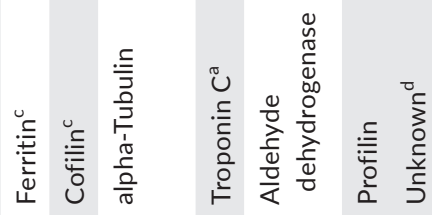

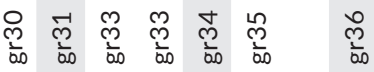

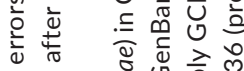

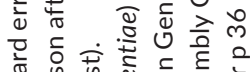

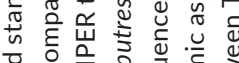

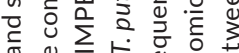

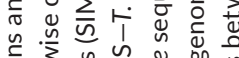

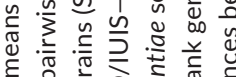

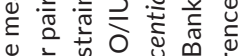

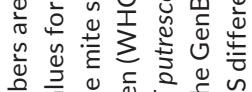

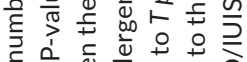

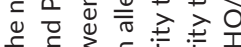

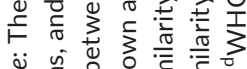

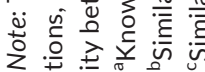




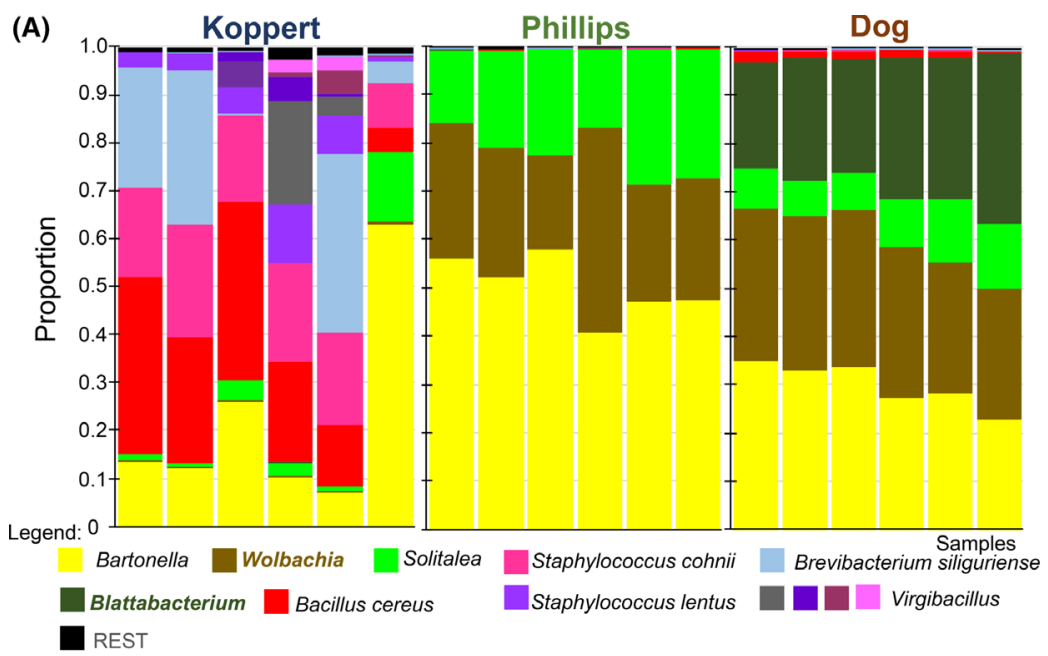

(B)
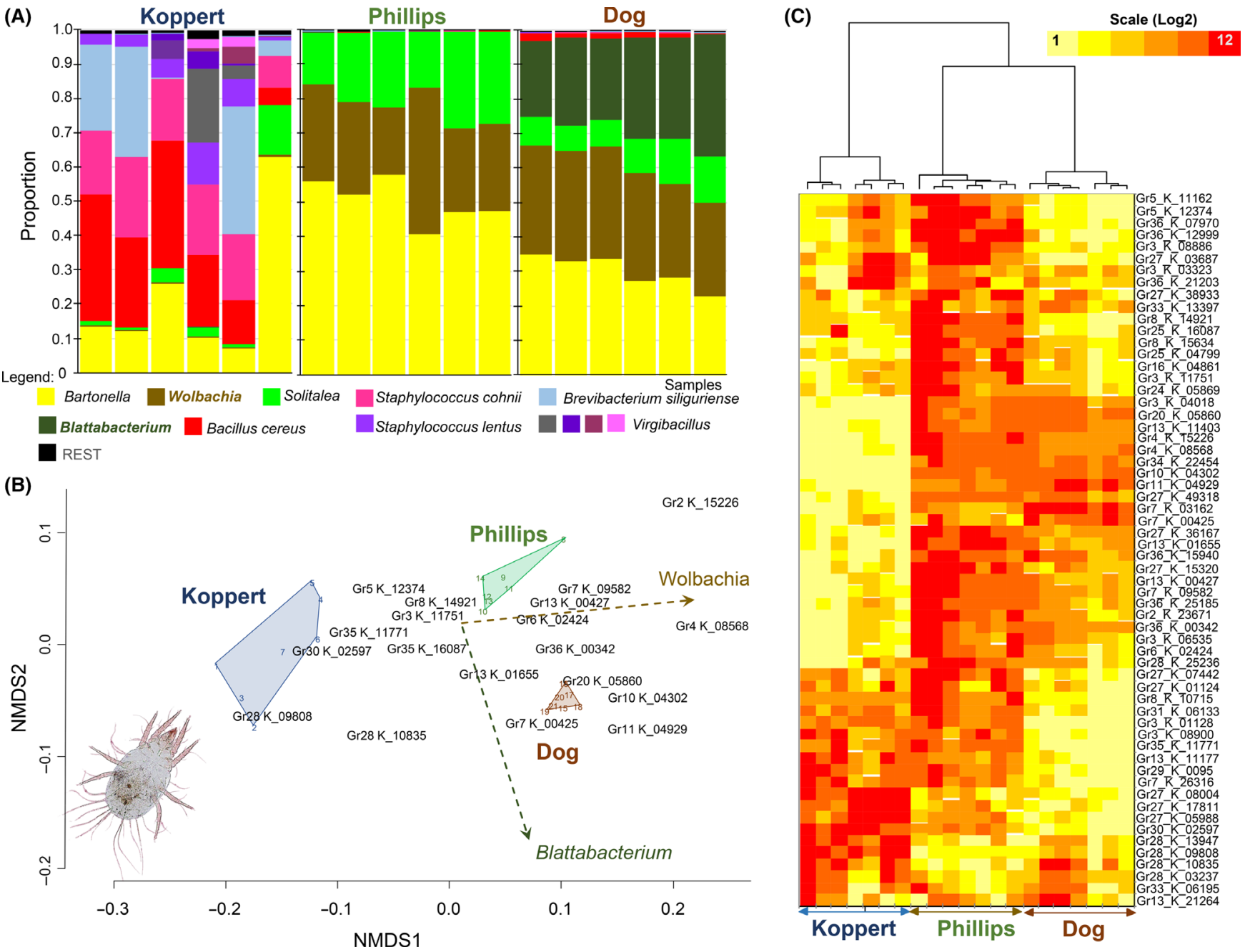

FIGURE 1 Allergen expression in three strains of the mite Tyrophagus putrescentiae with different microbiome compositions. The mite microbiomes were characterized based on V1-V3 16s RNA barcode sequencing (A). Differences in transcript expression among the mite strains were visualized by nonmetric multidimensional scaling (B); only sequences contributing to at least $75 \%$ of the total variability (SIMPER test) between the mite strains are shown. Differences in the transcript expression of selected immunogenic proteins are shown on the heatmap (C)

Until now, only eight allergens, Tyr p 2, 3, 10, 13, 28, 34, 35, and 36 , have been described in T. putrescentiae (IUIS/WHO allergen database). In this study, we investigated the expression of these allergens (and additional putative allergens predicted by us in silico) in three different strains of $T$. putrescentiae with known microbiomes. ${ }^{5}$ We used V1-V3 16S RNA sequence data from the three strains of mites. ${ }^{5}$ The transcriptomes were obtained by Illumina HiSeq 2500, and the raw reads were deposited in the SRA database (SUB4527480) (Table S1). The reads were processed and assembled in CLC Genomic Workbench v. 11 (Qiagen) (Tables S2-S4); the resulting assembly was then annotated by Prokka. ${ }^{7}$ The identified cDNA and protein sequences were compared to sequences of known mite allergens using BLAST, and the sequences with the lowest $\mathrm{E}$ values were compared manually to known and predicted allergen protein sequences (see Supplementary Material and methods).

We obtained 21 transcriptomes (seven biological replicates per every strain) and found 78 transcripts belonging to the eight known allergens of T. putrescentiae (see Supplementary Results); we also found 16 putative allergens based on sequence similarity with other mite species (groups 4-8, 11, 14, 16, 20, 24, 25, 27, 30, 31, 33, and 38; Table 1). ${ }^{8}$ Our BLAST search showed high similarity of our transcriptomes to some contigs of the genomic assembly of the fly Rhagoletis zephyria from GenBank (GCF_001687245.1). Several independent GenBank sequences of $T$ putrescentiae allergens also showed high identities (99\%-100\%) to the "R. zephyria" genome. These data suggest that the GenBank assembly of $R$. zephyria contains a significant portion of T. putrescentiae DNA (nearly $100 \mathrm{Mb}$ ), most likely due to inadvertent laboratory contamination. Such contamination is not surprising because $T$. putrescentiae commonly infects insect and fungal cultures in the laboratory. Nevertheless, the chimeric mite-fly GenBank "R. zephyria" genome facilitated our search for mite proteins (Table 1). We also detected polymorphisms in several allergens (Table 1). Coding sequences of some allergens were found in different contigs, for example, 2, 3, 5, 6, 7, 8, 13, 27, 28, 35, and 36 (Table 1).

In this study, we employ an approach to continue on a recent description of the allergen-producing mite microbiome. ${ }^{6}$ Lee et al $^{6}$ 
demonstrated that the microbiome profiles of Dermatophagoides farinae, Dermatophagoides pteronyssinus, and T. putrescentiae change after antibiotic treatment and bacteria that influence the endotoxin concentration. ${ }^{6}$ In this study, we set out to show that bacteria not only affect the endotoxin concentration but also their presence is correlated with the mite allergen expression level. The authors did not consider the intraspecies variability of the microbiome. For example, Solitalea-like (Sphingobacteriaceae_JN236497) and Bartonella-like bacteria (Bartonella_JX001274) form the microbiome of the Korean strain of T. putrescentiae. ${ }^{6}$ We previously observed that strains of T. putrescentiae are inhabited by unique microbial communities formed by intracellular (Wolbachia, Cardinium), putative intracellular (Solitalea, Blattabacteriumlike bacteria), and gut-associated symbionts (Bartonella-like bacteria, Bacillus sp.). ${ }^{5}$ All of these strains have unique and stable microbiome compositions with different proportions of the abovementioned bacterial taxa, suggesting a question of whether the allergen production of strains with different symbionts is similar or different.

Expression analyses indicated significant differences in transcript abundances among the three tested mite strains (ANOSIM perm. $=1000$; $R=0.9518, P<0.001)$. Among all the mite strains, the differences in allergen transcript expression levels were strong (Figure 1), as shown by nonmetric multidimensional scaling (NMDS; Table 1). The profiles of both intracellular symbionts (Wolbachia and Blattabacterium-like) significantly contributed $(P<0.001)$ to the explained variability of transcript expression after forward variable selection (Figure 1A).

In the two mite strains with intracellular bacterial symbionts (Phillips and Dog), many immunogenic proteins showed increased expression levels (Figure 1B), as shown by NMDS, where these groups were separated from the group lacking these bacteria along the x-axis: alpha-amylase (group 4), chymotrypsin (group 6), bactericidal-permeability increasing-like protein (group 7), tropomyosin (Tyr p 10), paramyosin (group 11), fatty acid-binding protein (Tyr p 13), arginine kinase (group 20), and an unknown group 36 allergen. In contrast, the Koppert population (no intracellular bacteria) had higher expression of allergen group 5 , heat shock protein (Tyr p 28), ferritin (group 30), and aldehyde dehydrogenase (Tyr p 35) than the mite populations having intracellular bacteria, Phillips and Dog (Figure 1). The Koppert population is connected to highly abundant Bacillus cereus, a bacterium previously shown to be associated with the feces of T. putrescentiae. ${ }^{9}$ The $y$-axis of our NMDS analysis separates the two latter populations: The Dog population is characterized by higher expression of bactericidal-permeability increasing-like protein (group 7), tropomyosin (Tyr p 10), paramyosin (group 11), and arginine kinase (group 20), while the relative expression of alpha-amylase (group 4), chymotrypsin (group 6), bactericidal-permeability increasing-like protein (group 7), and fatty acid-binding protein (Tyr p 13) is higher in the Phillips population. The increased expression of muscle proteins/ allergens (tropomyosin and paramyosin) and digestive enzymes (amylase and chymotrypsin) suggests a correlation with increased mite population growth. The growing mite cultures produce more juveniles that can contain higher muscle proportion in their bodies than adults due to reduced reproductive organs.
Previous experiments have demonstrated that the allergen expression in $D$. pteronyssinus is quantitatively and/or qualitatively influenced by mite development, sex, and environment. ${ }^{10}$ Here, we demonstrate that differences among mite strains are correlated with the presence and absence of intracellular symbionts in individuals taken from the same population. This sampling technique is used by the majority of mite allergen studies.

In conclusion, our results indicate that allergen expression is variable across different mite populations, and this phenomenon can be linked to differences in their microbiome compositions. It is possible that due to symbiotic microbes, the mite population in the natural conditions produces different levels of allergens, although belong to the same species of mites. However, establishing whether this relationship is causative or correlational requires further experiments. Mite microbiome composition appears to be an important factor that should be considered in allergen production.

\section{ACKNOWLEDGMENTS}

The authors are obligated to Martin Markovic for technical support. $\mathrm{JH}$ was supported by a project of the Ministry of Education, Youth and Sports of the Czech Republic (no. CZ.02.2.69/0.0/0.0/16_027/ $0008503), M N$ and TE by the Czech Science Foundation: GACR no. 19-09998S, BS was supported by project RO0418 of the Ministry of Agriculture of the Czech Republic. PBK was supported by the Russian Foundation for Basic Research (grant no. 18-04-01092) and by the Russian Science Foundation (grant no. 16-14-10109).

\section{CONFLICTS OF INTEREST}

The authors declare that they have no relevant conflicts of interest.

\section{KEYWORDS}

Bartonella, Blattabacterium, house dust mite, intracellular symbionts, stored product mite, Wolbachia

$$
\begin{array}{r}
\text { Jan Hubert } \\
\text { Marta Nesvorna } \\
\text { Pavel Klimov } \\
\text { 2,3 } \\
\text { Scot E. Dowd } \\
\text { Bruno Sopko } \\
\text { Tomas Erban }^{1}
\end{array}
$$

${ }^{1}$ Crop Research Institute, Prague, Czechia

${ }^{2}$ Department of Ecology and Evolutionary Biology, University of Michigan, Ann Arbor, Michigan

${ }^{3}$ Institute of Biology, University of Tyumen, Tyumen, Russia

${ }^{4}$ MR DNA (Molecular Research LP), Shallowater, Texas

Correspondence Jan Hubert, Crop Research Institute, Drnovska 507, Praha 6, Ruzyne, Prague, CZ-16106, Czechia. Email: hubert@vurv.cz 
ORCID

Jan Hubert iD https://orcid.org/0000-0003-0740-166X

Marta Nesvorna (iD https://orcid.org/0000-0003-0572-4131

Pavel Klimov (iD https://orcid.org/0000-0002-9966-969X

Scot E. Dowd iD https://orcid.org/0000-0002-6296-1427

Bruno Sopko iD https://orcid.org/0000-0002-5580-1871

Tomas Erban (D) https://orcid.org/0000-0003-1730-779X

\section{REFERENCES}

1. Trivedi B, Valerio C, Slater JE. Endotoxin content of standardized allergen vaccines. J Allergy Clin Immunol. 2003;111(4):777-783.

2. Valerio CR, Murray P, Arlian LG, Slater JE. Bacterial 16S ribosomal DNA in house dust mite cultures. J Allergy Clin Immunol. 2005;116(6):1296-1300.

3. Dzoro S, Mittermann I, Resch-Marat Y, et al. House dust mites as potential carriers for IgE sensitization to bacterial antigens. Allergy. 2018;73(1):115-124.

4. Kim JY, Yi M-H, Hwang Y, et al. 16S rRNA profiling of the Dermatophagoides farinae core microbiome: Enterococcus and Bartonella. Clin Exp Allergy. 2018;48(5):607-610.

5. Erban T, Ledvinka O, Nesvorna M, Hubert J. Experimental manipulation shows a greater influence of population than dietary perturbation on the microbiome of Tyrophagus putrescentiae. Appl Environ Microbiol. 2017;83(9):e00128-17.

6. Lee J, Kim JY, Yi M-H, et al. Comparative microbiome analysis of Dermatophagoides farinae, Dermatophagoides pteronyssinus, and Tyrophagusputrescentiae.JAllergy ClinImmunol.2019;143(4):1620-1623.

7. Seemann T. Prokka: rapid prokaryotic genome annotation. Bioinformatics. 2014;30(14):2068-2069.

8. Allergen Nomenclature. Astigmata. WHO/IUIS Allergen Nomenclature Sub-Committee; 2019. http://www.allergen.org/ search.php?TaxOrder=Astigmata. Accessed February 8, 2019.

9. Erban T, Rybanska D, Harant K, Hortova B, Hubert J. Feces derived allergens of Tyrophagus putrescentiae reared on dried dog food and evidence of the strong nutritional interaction between the mite and Bacillus cereus producing protease bacillolysins and exo-chitinases. Front Physiol. 2016;7:53.

10. Vidal-Quist JC, Ortego F, Lombardero M, Castanera P, HernandezCrespo P. Allergen expression in the European house dust mite Dermatophagoides pteronyssinus throughout development and response to environmental conditions. Med Vet Entomol. 2015;29(2):137-146.

\section{SUPPORTING INFORMATION}

Additional supporting information may be found online in the Supporting Information section at the end of the article.

\section{Inadequate knowledge of allergen immunotherapy among athletes with allergic rhinitis: A post hoc analysis}

To the Editor:

In our recent paper-based questionnaire survey of 636 German professional and hobby athletes, we observed a high prevalence of allergic rhinitis ( $n=269 ; 42.6 \%$ in total, $36.1 \%$ confirmed by a physician, $6.5 \%$ self-diagnosed). ${ }^{1}$ We presented evidence suggesting that this condition substantially impairs athletes' physical fitness. The negative impacts of allergic rhinitis on sport performance can be lessened by allergen immunotherapy (AIT), whereas symptomatic medications elicit only modest effects on sport performance and physical fitness in this particular group of allergic rhinitis sufferers. ${ }^{1}$

This post hoc analysis was conducted in the above-mentioned 269 athletes to better understand (a) athletes' knowledge of AIT, (b) the reasons why most athletes had not been treated with AIT, and (c) the demographic and diagnostic factors influencing the athletes' decision to undergo AIT.

Methods of the study and questionnaire are presented in Online Repository.

Although the majority of athletes (230 out of 265 athletes who responded to this question; $86.8 \%$ ) had "heard" about AIT, most (213; 80.4\%) were familiar with the conventional subcutaneous immunotherapy. The noninvasive route of administration with its better safety and tolerability profile was largely unknown to them: 57 out of 265 (21.5\%) of athletes were aware of sublingual immunotherapy with drops and 37 (14.0\%) with tablets. About half of the athletes (110 out of $265,41.5 \%$ ) knew about short-term subcutaneous immunotherapy comprising 4-7 injections per year. Even fewer athletes were familiar with pre-co-seasonal sublingual immunotherapy administered before and during the pollen season: 27 out of 265 (10.2\%) were aware of drops and 19 (7.2\%) of tablets.

The athletes' knowledge about AIT came mostly from physicians (69.7\%). Hardly any athletes obtained information from public channels (academic literature: 6.1\%; the Internet: 5.3\%; newspaper: 4.8\%; television/radio: 3.1\%; pharmacy: 3.1\%; German Allergy and Asthma Association: $1.8 \%)$. Only $15.4 \%$ of athletes evaluated their physician's consultation regarding AIT as being "good" (score 6-7), whereas $50.4 \%$ gave a "moderate" (score $3-5$ ) and $34.2 \%$ "poor" rating (score 1-2). These results mirrored the athletes' knowledge of AIT; only $8.3 \%$ of athletes rated their knowledge as being "good" (score $6-7$ ), $56.4 \%$ as "moderate" (score $3-5$ ), and $35.3 \%$ as "poor" (score 1-2). 